\title{
STING-associated vasculopathy with onset in infancy
}

INSERM

\section{Source}

INSERM. (1999). Orphanet: an online rare disease and orphan drug data base. STINGassociated vasculopathy with onset in infancy. ORPHA:425120

STING-associated vasculopathy with onset in infancy (SAVI) is a rare, genetic autoinflammatory disorder, type I interferonopathy due to constitutive STING (ST imulator of INterferon Genes) activation, characterized by neonatal or infantile onset systemic inflammation and small vessel vasculopathy resulting in severe skin, pulmonary and joint lesions. Patients present with intermittent low-grade fever, recurrent cough and failure to thrive, in association with progressive interstitial lung disease, polyarthritis and violaceous scaling lesions on fingers, toes, nose, cheeks, and ears (which are exacerbated by cold exposure) that often prog ress to chronic acral ulceration, necrosis and autoamputation. 